\title{
Comparative Study of Methods for Atrial Fibrillation Cycle Length Estimation in Fractionated Electrograms
}

\author{
Diego Osorio $^{1}$, Raúl Alcaraz ${ }^{2}$, José J Rieta ${ }^{1}$ \\ ${ }^{1}$ BioMIT.org, Electronic Engineering Department, Universitat Politecnica de Valencia, Spain \\ ${ }^{2}$ Research Group in Electronic, Biomedical and Telecomm. Eng., Univ. of Castilla-La Mancha, Spain
}

\begin{abstract}
Complex fractionated atrial electrograms (CFAEs) are electrograms (EGMs) characterized by a high variability both in amplitude and waveforms, making the estimation of their cycle length $(C L)$ a difficult task to perform. The $C L$ is a widely employed parameter for the characterization of the electrical activity within the atria, thus serving to guide catheter ablation, one of the most effective cardiac procedures for the treatment of AF nowadays. The present study aims to compare the performance of three different methods estimating the CL from a set of 50 recordings with CFAEs previously annotated by two expert physicians. The first algorithm is based on an adaptive amplitude threshold, while the second method performs the detection of the highest activations and a later detection of peaks within intervals longer than 1.5 times the median cycle length of the EGM. Finally, we introduce a novel hybrid method which performs a main amplitude detection, which is facilitated by the equalization of high and low amplitude activations in CFAEs according to a fractionation parameter, and a subsequent search for lower activations within intervals longer than the median $C L$, decreasing the amplitude threshold proportionally to the intervals' length. Our method outperformed the other analyzed detectors, which confirms that the method proposed is a more accurate estimator of atrial CL for CFAEs.
\end{abstract}

\section{Introduction}

Atrial fibrillation (AF) is the most common arrhythmia in clinical practice, affecting more than 20 million men and 12 million women worldwide. AF shows an estimated prevalence higher than $13 \%$ for individuals older than 80 years [1] and its prevalence is expected to increase in the forthcoming decades due to the aging of the population. Although the current economic impact of AF on the healthcare services is high, an annual incremental cost from $\$ 6$ billion to $\$ 26$ billion is estimated, only in the United States, for the next decades [2]. Since the physio- logical mechanisms responsible of the onset, maintenance as well as termination of AF are still not fully understood, this evidence highlights the need for significant improvements in AF research that could lead to tailored and more effective therapeutical strategies.

Catheter ablation (CA) is one of the most effective and employed cardiac procedures for the treatment of AF, and is expected to become the first-line therapy for AF rhythm control in the next 20 years [3]. This technique performs scars in the endocardium, through the application of highenergy radiofrequency signals, to prevent ectopic electrical activity from maintaining the AF. It has been reported that the pulmonary veins (PVs) are the main source of ectopic foci, provoking AF in $82 \%$ to $90 \%$ of patients with paroxysmal AF [4,5]. Furthermore, for those patients with persistent AF requiring additional ablation [6], the mapping and ablation of complex fractionated atrial electrograms (CFAEs) is a complementary strategy. Within this context, the atrial cycle length (CL) is defined as the time interval between two consecutive local activations waves (LAWs), and is a widely employed parameter to characterize atrial electrograms (EGMs). Since CFAEs can be defined as EGMs in which an average CL shorter than $120 \mathrm{~ms}$ is present [7], the computation of local as well as average $\mathrm{CL}$ is commonly employed for the guidance and ablation of AF beyond the PVs.

The objective of this study is to analyze the accuracy of three different LAWs detectors estimating the atrial CL of CFAEs, which may be employed in clinical practice to assist CA procedures. For this comparative study, two reference methods were employed: the adaptive amplitude threshold (AAT) method developed by Faes et al. [8] and the cycle length iterator (CLI) method introduced by $\mathrm{Ng}$ et al. [9]. In addition, a third novel LAWs detector has been introduced making use of a hybrid fractionation degree (HFD) approach [10]. The method performs LAW detection by considering both amplitude and CL of local waves together with an estimation of the fractionation degree of the EGM under study. 


\section{Methods}

\subsection{Electrograms dataset}

The dataset employed in this study consisted of a set of 50 recordings with CFAEs exported from a CardioLab system (General Electric, Wauwatosa, WI, USA) after written consent of patients undergoing CA procedures. EGMs were filtered out by a $0.5-500 \mathrm{~Hz}$ band-pass filter and a notch filtered at $50 \mathrm{~Hz}$ and resampled at $1 \mathrm{kHz}$. Next, the EGMs were denoised using a wavelet transform filtering since its application outperforms the results of regular filtering [11]. Finally, the local activation waves (LAWs) within the dataset were manually annotated by two expert physicians who, in addition, validated the presence of CFAEs as such, since the entire collection consisted of type III EGMs according to the classification proposed by Wells and coworkers [12].

\subsection{Adaptive amplitude threshold method}

The AAT method developed by Faes et al. in 2002 [8] is an algorithm based on the EGM preprocessing introduced by Botteron and Smith [13]. Moreover, it relies on an adaptive amplitude threshold estimated taking into account the last ten activations' amplitude with decreasing weights, so that the last activation weight is higher than the previous one, and so on. The algorithm begins with a $40-250 \mathrm{~Hz}$ band-pass filter, a rectification of the resulting signal, and a final low-pass filter with a cut-off of $20 \mathrm{~Hz}$. This preprocessing corresponds with the one introduced by Botteron in 1995, yielding the signal $s_{w}$. Later, this signal is employed as a reference for activations detection, controlled by a blanking period between activations of $55 \mathrm{~ms}$. In parallel, after the EGM rectification process, the output signal is filtered by a non-casual moving-average filter with 90 coefficients generating the signal $s_{f}$. Finally, the activations times are set on the positive zero crossings of signal $s_{f}$ which are closer to a detected peak within signal $s_{w}$ [8].

\subsection{Cycle length iterator method}

$\mathrm{Ng}$ et al. introduced in 2014 a LAWs detector for the estimation of CL in AF EGMs called the CLI method [9]. The preprocessing employed is similar to Botteron's, substituting the $40-250 \mathrm{~Hz}$ band-pass filter by a $40 \mathrm{~Hz}$ highpass filter and increasing the cut-off of the low-pass filter from 20 to $30 \mathrm{~Hz}$. This algorithm performs a gradual detection of the highest peaks setting a blanking period from each detected activation of $50 \mathrm{~ms}$. This detection process stops when the median CL of the EGM is lower than $275 \mathrm{~ms}$. In addition, a second condition has to be met: either the amplitude of the new detected peak is $20 \%$ less than the amplitude of the previous peak or the mean CL is less than $5 \mathrm{~ms}$ plus the median CL. The final stage of the detection is exclusively based on the length of the intervals between activations. For those intervals longer than 1.5 times the median $\mathrm{CL}$, the highest peak within the interval is selected as a new activation, repeating this process until there is no more long intervals [9].

\subsection{Hybrid fractionation degree method}

The HFD method based the detection process on a hybrid approach of a core amplitude detection, using both a blanking period between activations of $50 \mathrm{~ms}$ and a fixed amplitude threshold, as well as on a later detection of lower activations within intervals longer than the median CL [10]. The preprocessing performed is also similar to Botteron's, using the same implementation of the $20 \mathrm{~Hz}$ low-pass filter, so just the band-pass filter low cut-off is decreased from 40 down to $20 \mathrm{~Hz}$, since it has been observed that, with this configuration, those activations with a predominance of slow components were better processed, easing the later detection stage.

The detection stage begins with the main amplitude detection, which is facilitated by performing a reduction of the characteristic wide amplitude variability in CFAEs, which are selected as a function of the fractionation degree of each EGM [10]. After the amplitude detection, the median CL of the EGM is calculated from the current set of detected activations and taken as a reference value to analyze those intervals between activations longer than it. A reduction of the amplitude threshold proportional to the length of each interval longer than the median CL is applied with the aim to detect low amplitude activations that could have been lost. Finally, aiming to improve the detection precision and a further better estimation of the CL, the activation times are set on the barycenter of each LAW, computed as the value that divides the area of the modulus of the LAW into two equal parts [10]. Furthermore, should the estimation of the barycenter of two adjacent activations is located within an interval shorter than $50 \mathrm{~ms}$, the activation with the lowest amplitude is discarded from the set of detected activations [10].

\subsection{Evaluation of CL estimation}

The performance of each method as estimator of CL was evaluated as the absolute difference between the mean CL, from the manual annotations in the database, and the mean CL reported by each evaluated method when applied on the set of EGMs. Then, the global error committed by each method for the entire dataset was calculated in terms of the mean and the standard deviation of the detection errors. 


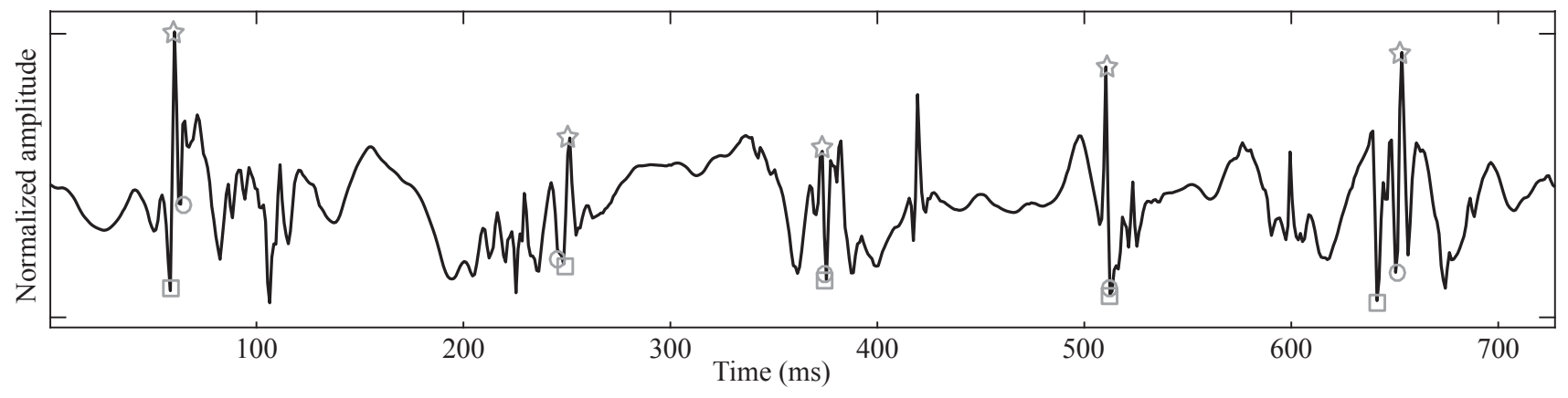

Figure 1. Comparative example of the activation times estimation for each method over a denoised type III EGM. The circles correspond with the activation times estimated for the adaptive amplitude threshold (AAT), the squares are the estimations for the cycle length iterator (CLI) and the stars refer to the hybrid fractionation degree (HFD) method.

\section{Results}

\subsection{Preprocessings comparison}

These output signals from the preprocessings present proportional waveforms to the amplitude of the EGM components, with frequencies between the cut-offs of the bandpass filter of each method. Thus, the filtering performed in the HFD method $(20-250 \mathrm{~Hz})$ has provided higher amplitudes for slow activations. This preprocessing has performed a similar response for fast activations than the other methods, but dealing better with slower activations which, combined with the amplitude variability reduction for CFAEs, significantly facilitates or even enables their later detection.

\subsection{CL estimation results}

Results of the mean CL estimation for each method are shown in Figure 2. The errors correspond with the absolute difference between the mean CL of the manual annotations and the mean CL estimated by each method. For the AAT, CLI and HFD methods, the CL estimation error computed for the whole dataset were $19.83 \pm 14.44 \mathrm{~ms}$, $12.11 \pm 11.01 \mathrm{~ms}$ and $5.66 \pm 4.19 \mathrm{~ms}$, respectively. Results show a better performance for the HFD method, committing approximately half of the mean error made by the CLI method, while the AAT method presents the highest error values, both for the mean and the standard deviation. Figure 1 shows the performance of each method for the estimation of the activations times over an example type III EGM. The causes that provoke the CL estimation results obtained by each method are both the innacuracy in the estimation of the activations times and, to a greater extent, the detection errors committed.

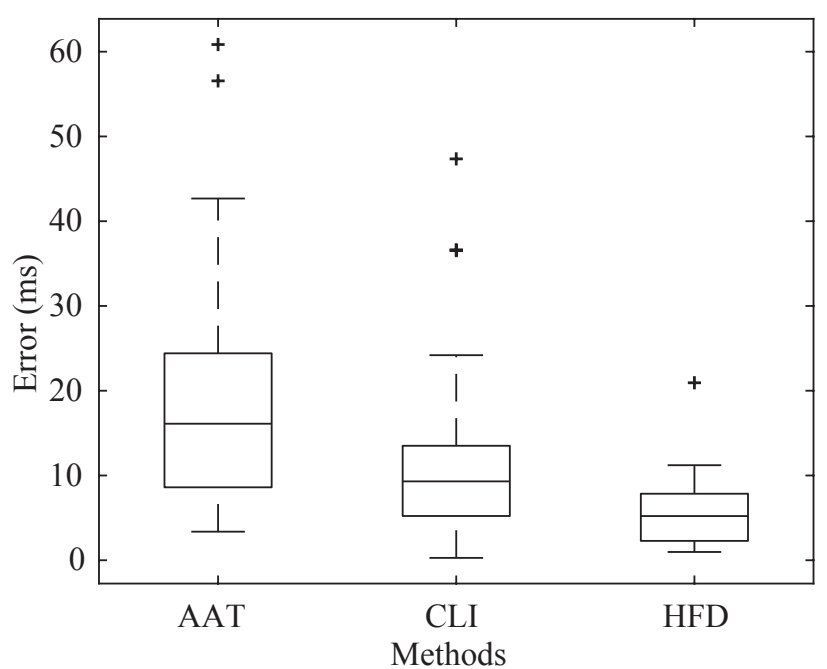

Figure 2. Performance comparison of the three analyzed methods. Absolute errors committed in estimating the mean CL in CFAEs with respect to manual annotations are presented.

\section{Discussion}

The HFD method has demonstrated to be a more accurate estimator of CL for CFAEs than the other two methods analyzed. The hybrid detection approach, together with the reduction of the amplitude variability in CFAEs and the modification of the Botteron's cut-off frequencies, have shown to perform better in the estimation of CL. Furthermore, the use of the barycenter of each LAW to define the activation times improves the precision of their location and, thus, serves to estimate more precisely the CL. Both the insufficient response of Botteron's preprocessing to slow activations together with the high amplitude variability of CFAEs explain the detection errors committed by the AAT method due to a poor adaptation of its amplitude threshold. 
However, although the CLI and AAT strategies perform a similar preprocessing, the influence in the results of this insufficient response to CFAEs amplitude variations is less severe for the CLI method. This could happen because the detected activations with the CLI are always the highest peaks, regardless of their absolute value, and also the final detection of activations by their position ensures a robust detection. Finally, the preprocessing of the HFD method facilitates both the main amplitude detection and the later detection of activations within long intervals, since they are also detected by an amplitude threshold proportional to the interval length.

\section{Conclusions}

The HFD method has demonstrated to be a more suitable estimator of CL to be employed for the characterization of the atrial electrical activity to assist CA procedures, since it has shown precise results even for highly fractionated electrograms, which are the most difficult to deal with. Therefore, this guidance may serve to find more accurately possible ablation targets responsible of the AF maintenance to improve the outcomes of the procedure.

\section{Acknowledgements}

Research supported by grants TEC2014-52250-R and DPI2017-83952-C3 MINECO/AEI/FEDER, UE.

\section{References}

[1] Kirchhof P, Benussi S, Kotecha D, Ahlsson Aea. 2016 ESC Guidelines for the management of atrial fibrillation developed in collaboration with EACTS. European Heart Journal 2016;37(38).

[2] Pathak RK, Evans M, Middeldorp ME, Mahajan R, Mehta AB, Meredith M, Twomey D, Wong CX, Hendriks JML, Abhayaratna WP, Kalman JM, Lau D, Sanders P. Costeffectiveness and clinical effectiveness of the risk factor management clinic in atrial fibrillation: The CENT study. JACC Clinical Electrophysiology 2017;3(5):436-447.

[3] Gillis AM, Krahn AD, Skanes AC, Nattel S. Management of atrial fibrillation in the year 2033: New concepts, tools, and applications leading to personalized medicine. Canadian Journal of Cardiology 2013;29(10):1141-1146.

[4] Lau DH, Schotten U, Mahajan R, Antic NA, Hatem SN, Pathak RK, Hendriks JML, Kalman JM, Sanders P.
Novel mechanisms in the pathogenesis of atrial fibrillation: Practical applications. European Heart Journal 2016; 37(20):1573-1581.

[5] Morin DP, Bernard ML, Madias C, Rogers PA, Thihalolipavan S, Estes III NAM. The state of the art: Atrial fibrillation epidemiology, prevention, and treatment. Mayo Clinic Proceedings 2016;91(12):1778-1810.

[6] Verma A, Jiang C, Betts TR, Chen J, Deisenhofer I, Mantovan R, Macle L, Morillo CA, Haverkamp W, Weerasooriya R, Albenque JP, Nardi S, Menardi E, Novak P, Sanders P, STAR AF II Investigators. Approaches to catheter ablation for persistent atrial fibrillation. The New England Journal of Medicine 2015;372(19):1812-1822.

[7] Baumert M, Sanders P, Ganesan A. Quantitativeelectrogram-based methods for guiding catheter ablation in atrial fibrillation. Proceedings of the IEEE 2016; 104(2):416-431.

[8] Faes L, Nollo G, Antolini R, Gaita F, Ravelli F. A method for quantifying atrial fibrillation organization based on wave-morphology similarity. IEEE Transactions on Biomedical Engineering 2002;49(12 I):1504-1513.

[9] Ng J, Sehgal V, Ng JK, Gordon D, Goldberger JJ. Iterative method to detect atrial activations and measure cycle length from electrograms during atrial fibrillation. IEEE Transactions on Biomedical Engineering 2014;61(2):273-278.

[10] Osorio D, Alcaraz R, Rieta JJ. A fractionation-based local activation wave detector for atrial electrograms of atrial fibrillation. In Computing in Cardiology Conference (CinC), volume 44. IEEE, 2017; In press.

[11] Martínez-Iniesta M, Ródenas J, Alcaraz R, Rieta JJ. Waveform integrity in atrial fibrillation: The forgotten issue of cardiac electrophysiology. Annals of Biomedical Engineering 2017;45(8):1890-1907.

[12] Wells Jr JL, Karp RB, Kouchoukos NT, MacLean WAH, James TN, Waldo AL. Characterization of atrial fibrillation in man: Studies following open heart surgery. Pacing and Clinical Electrophysiology 1978;1(4):426-438.

[13] Botteron GW, Smith JM. A technique for measurement of the extent of spatial organization of atrial activation during atrial fibrillation in the intact human heart. IEEE Transactions on Biomedical Engineering 1995;42(6):579-586.

Address for correspondence:

Diego Osorio

Electronic Engineering Department, Building 7F

Universidad Politécnica de Valencia

Camino de Vera, s/n, 46022, Valencia, Spain

E-mail: dosorio@upv.es 